\section{Evaluación de un programa de política social: Programa Materno Infantil y Nutrición}

\author{
Evaluation of a social policy program: \\ the Maternal and Child Health and Nutrition Program
}

Alicia Aronna ${ }^{1}$

\title{
Introducción
}

1 Instituto de la Salud Juan Lazarte, Universidad Nacional de Rosario, Rosario, Argentina.

Correspondencia A. Aronna

Instituto de la Salud Juan Lazarte, Universidad

Nacional de Rosario.

9 de Julio 1590, 5o Piso, 2000

Rosario, Argentina.

gosende@sinectis.com.ar

\section{Abstract}

This study is based on an evaluation of the Maternal and Child Health and Nutrition Program (PROMIN) targeting pregnant women and their children under five years of age. The objective was to identify the conditioning factors for the Program's implementation in Rosario, Argentina. There were three levels of analysis: the organizational environment as perceived by the Executive Directors of the Health and Child Development Centers; management of interventions by the health teams; and the community's perception of the program's accessibility and acceptability. Two centers were chosen for the year 1998. Empirical evidence was obtained through quantitative and qualitative procedures. The results suggest that the two centers' respective organizational environments influenced the intervention strategies. The goal for coverage had been set at $80 \%$. Documentation of the interventions by the two teams shows a partial and heterogeneous implementation. In terms of accessibility, mothers recognize the institutions by their reputation, quality of services, and extra services beyond the PROMIN basics. Acceptability is expressed as the provision of supplementary nutrition.

Public Policy; Program Evaluation; Maternal and Child Health
La evaluación de las intervenciones implementadas en el sector salud, basada en principios epidemiológicos, debe ser considerada como una práctica indispensable, cuyos resultados pueden y deben ser aplicados para facilitar la toma de decisiones en la búsqueda de estrategias de mayor efectividad, y a su vez, para asegurar los mecanismos que orienten el grado de cumplimiento de los objetivos propuestos, esto es, comprobar en qué medida se ha llegado a la población destinataria de las acciones previstas y a posteriori si se están mejorando efectivamente las condiciones de partida de estos grupos, según la visión y perspectiva de quien desarrolle la evaluación 1 .

En la medida que el proceso de trabajo del sector salud implica necesariamente la efectiva participación del beneficiario para la obtención de un servicio, no es posible la evaluación del proceso sin su participación por ser el destinatario/receptor de las acciones, ya que son quienes le van a dar la forma particular, en un momento y en un contexto dado, expresando cuan adecuadas y apropiadas fueron ejecutadas estas acciones.

Tanaka 2 indica que la eficacia de las actividades desarrolladas en un programa son mejor evaluadas con estudios o investigaciones particulares con una finalidad específica, estableciendo la separación entre actividad evaluativa de seguimiento y la investigación evaluativa. 
Contandriopoulos et al. 3 (p. 2) es más extensivo en sus conceptos y en los espacios donde desarrollar esta forma de planteamiento: "evaluar consiste fundamentalmente en acercar un juicio de valor sobre una intervención o sobre no importa cual de sus componentes, con el propósito de ayudar a la toma de decisiones. Este juicio puede resultar de la aplicación de criterios y de normas (evaluación normativa) o elaborarse a partir de un recorrido científico (investigación evaluativa)".

Tal investigación evaluativa, que consiste en hacer un juicio ex post sobre la intervención, puede indagar la pertinencia, los fundamentos teóricos, la productividad, los efectos y el rendimiento de una intervención, así como las relaciones existentes entre la intervención y el contexto en la que es situada, con el propósito de ayudar a la toma de decisiones.

En este sentido, el análisis de implantación de un programa constituye un tipo de evaluación, cuyo objetivo consiste en identificar los procesos implicados en la producción de los efectos de una intervención en términos de una mejoría en el estado de salud de las poblaciones objetivo 4 .

Este modelo se aleja de aquéllos en los que sólo se apela a la observación de los efectos (caja negra), ya que resultan poco útiles para la mejora del diseño e implementación de las intervenciones. Se debe ir más allá del juicio de la eficacia de una intervención, indagando en torno a los factores condicionantes/explicativos de los resultados obtenidos 5 .

Denis \& Champagne 6 (p. 57) proponen una definición de los estudios de implantación superadora a la realizada por otros autores a quienes citan en especial 7, ellos explicitan: "un análisis de implantación se interesa en el estudio de los determinantes y en la influencia en la variación de la implantación en los efectos producidos por la intervención. Se sostienen conceptualmente en el análisis de la influencia sobre tres componentes: a) los condicionantes /determinantes contextuales en el grado de implantación de las intervenciones; b) las variaciones de la implantación en su eficacia; c) la interacción entre el contexto de implantación y la intervención en los efectos observados".

Con este marco se presenta una evaluación de un programa social desarrollado en algunos servicios de salud del subsector público del Municipio de Rosario, Argentina, cuyos resultados pueden servir como argumento racional y empíricamente fundamentado ante otros procesos de implantación que puedan suscitarse, mediando la decisión política de iniciar una intervención de este orden.

\section{Características generales del programa social}

El Programa Materno Infantil y Nutrición - Subproyecto Rosario 8 (PROMIN) se implementó en la Ciudad de Rosario como consecuencia del ofrecimiento realizado en el año 1992 por UNICEF Argentina a las autoridades municipales. Se instaló como consecuencia de un préstamo del BIRF(Banco Mundial), que el gobierno nacional asumió.

La implementación del PROMIN presentó una situación particular por coexistir en la ciudad servicios de salud de dependencia provincial y municipal, respectivamente.

El criterio de focalización de la población como estrategia inicial para determinar los destinatarios de las acciones (población NBI) y el establecimiento de normas para las actividades ubican a este programa en la línea de la planificación normativa.

El PROMIN de carácter integrador, promovía la articulación de las acciones de salud, promoción social y educación para mejorar la calidad de vida de madres y niños, optimizar la utilización de los recursos y la gestión operativa a través de la coordinación y planificación conjunta de los organismos involucrados.

Los objetivos propuestos fueron disminuir la morbimortalidad materna e infantil a través de la focalización y el mejor diseño, aplicación y coordinación de los servicios y programas de salud, nutrición, alimentación complementaria y desarrollo infantil; promover el desarrollo psicosocial de los niños entre 2 y 5 años y mejorar la eficacia y eficiencia de los programas de comedores escolares vigentes.

Las áreas componentes del PROMIN fueron: Salud, Desarrollo Infantil, Nutrición, Monitoreo y Evaluación; su duración se estableció en tres años.

\section{Propuesta evaluativa}

La propuesta evaluativa se sustentó en los siguientes ejes problemáticos: la visualización de diferencias entre el contenido programático, la ejecución de las acciones y la escasa participación de los equipos de salud locales; la necesidad de contar con resultados de estudios particularizados de las unidades socio-territoriales de PROMIN Rosario; el rol limitado que desempeñó el componente de monitoreo y evaluación del programa en la búsqueda de hallazgos que facilitaran la toma de decisiones; las deficiencias detectadas en el sistema de registro y en los procedimientos empleados, in- 
dicativos de una escasa adhesión programática y la necesidad de incorporar a la población como actor, para que pudiera dar cuenta en qué medida se sentían beneficiados y de qué modo lo expresaban.

Desde el marco conceptual de Contandriopoulos et al. 3 y Denis \& Champagne 6 , se realizó un análisis de implantación de PROMIN poniendo en juego tres niveles de análisis: la visión desde los equipos de salud, representados por los jefes de los Centros de Salud seleccionados, a los que se les requirió la identificación de los factores contextuales, (estructurales y organizativos), por ellos percibidos retrospectivamente en el proceso iniciado a partir de la ejecución de las acciones programáticas en Rosario (1996); un segundo nivel es el estudio del programa a través de la eficacia de los resultados, obtenidos en función del cumplimiento de las normas, cobertura y utilización de los servicios en un grupo particular, como son los niños desnutridos y las embarazadas. El tercer nivel de análisis se conforma con las representaciones de la población en torno a la accesibilidad y aceptabilidad del programa para recuperar en profundidad aspectos no programáticos.

Estos niveles analizados de forma articulada e interdependiente permitieron plantear el siguiente esquema de relaciones: el ambiente organizacional (factores contextuales) condicionó las estrategias de intervención desarrolladas desde los servicios, que a su vez se interrelacionan con las representaciones construidas por la población en términos de accesibilidad y aceptabilidad a este programa según componentes socioculturales.

El análisis contextual se concibe como los diferentes medios de implantación referidos a la adhesión al proyecto, la coherencia entre el programa y las actividades habituales del servicio de salud por parte de la organización seleccionada. El análisis de la influencia de la interacción entre el programa y el contexto permite el reconocimiento de los factores contextuales susceptibles de contribuir a la realización del potencial de la intervención en cuestión 6 .

El análisis de los efectos se estudió a través de la eficacia de los resultados, en los beneficiarios, entendiéndolos como los beneficios observados al aplicar un procedimiento, realizando la evaluación en términos del proceso de gestión que da lugar a la captación y seguimiento de la población. Este proceso está conformado por una serie de estrategias de intervención que se llevan a cabo por y entre profesionales y pacientes, vistas como comportamientos normativos que contribuyen a alcanzar ciertos logros 9 .
Las representaciones aparecen como formas de conocimiento social que permiten interpretar la realidad cotidiana; es un conocimiento socialmente elaborado y compartido. En el discurso espontáneo las representaciones aparecen organizadas en términos de conocimientos, valores, actitudes, creencias y opiniones que se manifiestan como un todo complejo sin diferenciación (Moscovici, 1961, apud Jodelet 10$)$.

Las representaciones de quienes resultaron beneficiarios de las acciones programadas permitieron recuperar desde otro nivel de observación, cuestiones que tuvieron que ver por un lado con la accesibilidad, es decir, con el proceso de buscar y recibir atención, lo que significa una gama continua de fenómenos pertenecientes al proceso de atención médica, donde en un extremo está la disponibilidad, que constituye la presencia física de los recursos para la salud y en consecuencia su capacidad para producir servicios y en el otro el acceso/la utilización, comprendiendo las características del recurso que facilitan o dificultan el uso por parte de los usuarios potenciales 11 .

La aceptabilidad, resultó de utilidad para analizar las percepciones subjetivas del acceso, las opiniones que el usuario tiene sobre la atención y las expectativas personales, con la satisfacción como componente inherente.

La investigación se llevó a cabo teniendo como objetivo general evaluar el nivel de implantación del PROMIN a través del análisis del proceso de gestión y resultados en términos de la accesibilidad y aceptabilidad en dos servicios de salud, durante el año 1998 que es el año de realización del estudio - la recogida de la información -, a tal año se refieren los resultados correspondientes al primer objetivo. Mil novecientos noventa y seis (1996) es el año de inicio del proceso de implantación del programa en los servicios de salud de Rosario; este proceso se pretende evaluar a partir de la perspectiva de los entrevistados, para cumplimentar el segundo y tercer objetivo.

En tanto que los objetivos específicos propuestos fueron: establecer (a) la cobertura alcanzada y las estrategias de intervención desde los servicios afectados a PROMIN, en relación con la captación y seguimiento de las embarazadas y niños menores de seis años, en particular los desnutridos; (b) el ambiente organizacional (los factores contextuales) en el desarrollo del proyecto desde la perspectiva de los jefes de las unidades de observación tomadas en cuenta (Centros de Salud y Centros de Desarrollo Infantil - CDI) y (c) la accesibilidad y la aceptabilidad desde las representaciones de la 
población beneficiaria según componentes socioculturales.

\section{Metodología}

El análisis de la implantación se llevó a cabo bajo la forma metodológica de estudios de casos múltiples con niveles de análisis interrelacionados. Se examinó el conjunto de las relaciones existentes entre las diferentes variables y categorías definidas para comprender el comportamiento de un fenómeno complejo que no puede ser aislado de su contexto 6,12. Se apeló a múltiples fuentes de información, tanto cualitativas como cuantitativas para lograr una mayor fiabilidad de los datos utilizados 13 . La potencia analítica depende de la profundidad del análisis realizado y no del número de unidades.

Se seleccionaron dos Centros de Salud Sur y Policial con sus respectivos CDI: Gurí y Jardín № 37 que iniciaron las acciones de PROMIN en marzo de 1996, ubicados en la zona sur de la Ciudad de Rosario y con dependencia municipal y provincial respectivamente, como forma de controlar comportamientos particulares de distintas administraciones.

Las estrategias metodológicas seleccionadas fueron:

a) Abordaje cuantitativo para la evaluación de la cobertura y estrategias de intervención, con relación a la captación y seguimiento de niños menores de 6 años desnutridos y embarazadas, llevada a cabo a través del método de trazadores elaborado por Kessner et al. 14 .

Se definió cobertura como la medida en que se llega a la población destinataria del proyecto en sus distintos agrupamientos de edad; y como estrategias de intervención en las actividades - normas y procedimientos de atención explicitadas en el proyecto considerando los componentes Salud, Nutrición y Educación.

La población objeto de estudio fueron los niños desnutridos menores de 6 años en los Centros de Salud Sur (181) y Policial (61) y las embarazadas 97( Sur) y 74 (Policial), captados entre marzo de 1996 y marzo de 1998, con domicilios en las respectivas zonas de focalización.

Los indicadores para la condición trazadora desnutrición en niños por grupos de edad fueron: (i) concordancia en la asistencia real a control y la norma de controles; (ii) concordancia entre la asistencia brindada y los procedimientos propuestos por el proyecto (utilización de curvas de crecimiento y desarrollo); (iii) porcentaje de recuperación por edad y por tipo de desnutrición; (iv) porcentaje de derivaciones a CDI de desnutridos de 2-5 años reali- zadas; (v) porcentaje de concurrencia a CDI de desnutridos por historia clínica; (vi) porcentaje de niños desnutridos institucionalizados (CDI) por concordancia de registros; (vii) porcentaje de referencias y contrarreferencias realizadas en pacientes desnutridos; (viii) porcentaje de referencias identificadas según lugar de derivación; (ix) porcentaje de inclusión de hoja de referencia y contrarreferencia en historia clínica; $(x)$ concordancia en la entrega de complementación alimentaria por edad; (xi) concordancia entre Centros de Salud y CDI en relación con el número y tipo de desnutridos; (xii) tiempo de permanencia de los desnutridos en CDI; y (xiii) controles por patología.

Para la condición trazadora embarazo: (i) porcentaje de captación de embarazadas; (ii) porcentaje de referencias y contrarreferencias realizadas en embarazadas; (iii) porcentaje de referencias identificadas según lugar de derivación; (iv) porcentaje de inclusión de hoja de referencia y contrarreferencia en historia clínica; (v) porcentaje de embarazadas captadas tempranamente; (vi) concordancia entre la asistencia brindada y los procedimientos propuestos por el proyecto (utilización de curvas de Rosso Mardones); (vii) concordancia en la entrega de complementación alimentaria; (viii) porcentaje de embarazadas según estado nutricional; (ix) cumplimiento de las visitas programadas (seguimiento); (x) porcentaje de embarazadas con patología positiva; (xi) porcentaje de embarazadas con cinco controles o más; (xii) porcentaje de embarazadas referidas para el control de puerperio desde las maternidades; (xiii) porcentaje de embarazadas con control de puerperio de acuerdo a programa; (xiv) porcentaje de embarazadas con FUM y peso inicial indicado en historia clínica; (xv) porcentaje de embarazadas con indicación de laboratorio; (xvi) porcentaje de embarazadas con indicación de ecografía; (xv) porcentaje de embarazadas según tipo de complementación alimentaria entregada; (xvi) lugar y tipo de parto en las embarazadas captadas; (xvii) porcentaje de derivación a otros servicios de salud; (xviii) porcentaje de historia clínica de embarazadas con indicación del peso del recién nacido.

La información se relevó a través de fuentes secundarias tales como el Documento Promin con las normas y procedimientos de atención, las historias clínicas de los Centros de Salud, las planillas de los CDI y los registros propios del programa.

Para el volcado y procesamiento de la información se diseñaron bases de datos cuya unidad de registro fueron los niños menores de 6 años desnutridos y las embarazadas; se obtu- 
vieron los resultados en función de los indicadores definidos y según el mayor o menor cumplimiento de las actividades. Se utilizaron los programas Epi Info 6.0 (Centers for Disease Control and Prevention, Atlanta, Estados Unidos) y SPSS/PC (SPSS Inc., Chicago, Estados Unidos). b) Abordaje cualitativo 15 para visualizar la evaluación desde otro ángulo y poder reinterpretar los resultados obtenidos con la aplicación del método de trazadores e incorporar la perspectiva de otros actores (los jefes de centros y los beneficiarios) y de este modo recoger a través de sus representaciones el grado de aceptación y el nivel de ajuste de PROMIN a sus expectativas particulares.

Esto significó la identificación de los factores contextuales percibidos por los jefes de centros en aquel momento y cómo ellos visualizaron los cambios y transformaciones que se fueron produciendo entre 1996 y 1998, es decir, aspectos organizacionales como la responsabilidad en las decisiones, la práctica asistencial concreta a partir de las acciones propuestas por el programa y la participación de los equipos en las metas; los aspectos estructurales son las características propias y naturales del programa, formas de implantación y la conformación de los equipos técnicos gerenciadores. Se intentó recuperar la marcha, los deseos, las prioridades y los cambios desde la perspectiva de los representantes de los equipos de salud 16 .

En cuanto a las valoraciones de las madres, también se instó a que recuperaran sus percepciones sobre el devenir de su vinculación con el programa en términos de la accesibilidad y aceptabilidad.

Se asumió la accesibilidad como la posibilidad de obtener atención fácil y oportuna cuando se necesita 9 ; conocer las instancias seguidas para la solución de los problemas de saludenfermedad de embarazadas y niños menores de 6 años de la zona de focalización, en particular la demanda a los servicios y la concreción de la atención; los obstáculos en el logro de la atención (disponibilidad de turnos, horarios, profesionales de preferencia, existencia de complementación alimentaria, derivación a CDI) y las dificultades en la relación población objetivo/personal de los servicios, que entorpecen el diagnóstico, utilización adecuada de los recursos terapéuticos disponibles, el seguimiento del paciente y la continuidad de la terapia aconsejada (en Centros de Salud y CDI).

La aceptabilidad de las actividades del programa, referente subjetivo y valorativo permitió deducir aspectos que daban cuenta de la efectividad de la atención, de los atributos de la relación médico-paciente en relación con las acciones desarrolladas en el servicio. En síntesis las representaciones de la población que pudieran dar cuenta acerca del grado de ajuste 17 entre las acciones desarrolladas por los servicios y las expectativas de los pacientes y sus familias. Es decir valorar la adaptación a la intervención, así como la visión global respecto al programa desarrollado en los servicios a los que concurrían. La satisfacción se consideró un componente inherente de la aceptabilidad.

La técnica de entrevista se aplicó a los cuatro jefes de centros por un lado y por otro a veinte madres de niños desnutridos, no desnutridos y embarazadas (diez por cada centro), buscando recuperar el status de la palabra 18 , la charla individual e intimista tomada como fuente de información de aspectos relacionados con los referentes temáticos.

Los resultados expuestos a continuación constituyen una síntesis de los hallazgos obtenidos; para facilitar la comunicación se obvian las transcripciones de los aportes realizados por cada uno de los entrevistados desde su lugar de actor particular.

\section{Resultados}

\section{De la cobertura alcanzada y de las estrategias de intervención}

La cobertura pediátrica, global y por edad, es diferencial: Sur aparece superando la meta en todos los grupos (100\%), mientras que Policial está alrededor del $54 \%$.

La cobertura alcanzada en embarazadas no alcanza la meta propuesta por PROMIN (80\%), la distancia para el cumplimiento es distinta en los dos espacios, Sur muestra un valor muy cercano al estipulado (78\%), no así Policial (55\%).

La desnutrición en la infancia es uno de los indicadores de criticidad y riesgo social; la población en Sur, aparecería en una situación de mayor vulnerabilidad relativa respecto de Policial $(14,5 \% / 11,9 \%$ versus $<10 \% / 10 \%$ para menores de 2 años y de 2-5 años) correlacionado con un mejor cumplimiento de las citaciones de seguimiento de crecimiento y desarrollo en Sur.

Para los desnutridos la norma establecía un control quincenal, el registro del seguimiento resultó adecuado en Sur 88,9\% y en Policial $82 \%$, expresando adhesión a la propuesta de los servicios. La ausencia de seguimiento constituye una llamada de atención por la falta de concurrencia a las citaciones: $7,2 \%$ y $11,5 \%$.

Más del 90\% de los desnutridos de ambos centros registró patologías asociadas (respiratorias y de la piel fundamentalmente) con una 
alta variabilidad en el número de controles (rango 0-23/0-15).

La entrega de la complementación alimentaria alcanzó $49,7 \%$ de adecuación a las normas en Sur y $57,4 \%$ en Policial.

La recuperación de los desnutridos, núcleo de interés en el marco de los objetivos programáticos y de las acciones específicas a desarrollar en ambos Centros de Salud, se registraron cifras por debajo del $40 \%$ (26,3\% Sur y $37,7 \%$ Policial). La constancia de la derivación de los desnutridos al respectivo CDI no superó el $13,2 \%$ ( $10 \%$ Sur y $13,2 \%$ Policial) y sólo en la mitad se especifica la concurrencia.

Del análisis de los registros surgen dificultades en el nivel de la articulación entre Centros de Salud y CDI, según se desprende del escaso número de niños cuyos nombres aparecen en los registros realizados por ambas instituciones (39,7\% Sur y 11,5\% Policial).

Sólo para Sur se obtuvo la mediana de institucionalización en CDI de ocho meses $\left(Q_{1}=3\right.$, $\mathrm{Q}_{3}=10$ ), tiempo escaso para producir cambios en los desnutridos.

La inclusión de las curvas para la valoración del estado nutricional de los niños se verificó en el $100 \%$ de las historias en ambos centros, no así la inclusión de la curva de Rosso Mardones, introducida por el programa, en particular en Policial 2,7\%, no así en Sur 91,7\%.

La consolidación de la red no se verificó, las referencias registradas alcanzaron el $41,4 \%$ Sur y $8,2 \%$ Policial de las derivaciones; las contrarreferencias sólo se consignaron un $34,7 \%$ y se incluyó la hoja de referencia y contrarreferencia sólo en Sur 5,3\%.

En ambos centros se respetó el circuito de derivaciones establecido por programa con bajo registro, aún cuando las mismas fueron escasas.

Para las embarazadas, la captación fue buena en ambos centros $(85,1 \% / 70,5 \%)$, con guarismos más alentadores en Policial para su seguimiento $(72,2 \% / 81,1 \%)$, captación temprana (75\%/84\%) y con cinco controles o más $(60,8 \% /$ $75,7 \%)$.

El estado nutricional normal representó algo más de la tercera parte del grupo $(36,1 \% /$ $41 \%$ ) y el sobrepeso y la obesidad concentraron el $32,9 \%$ y $36,1 \%$, posible expresión de malos hábitos alimentarios. Son mujeres con patologías asociadas en un $67 \%$ en Sur y $37,8 \%$ en Policial (HTA, infección urinaria, anemia). El registro de la solicitud de laboratorio $(90 \% / 81,1 \%)$ y ecografías $(89,7 \% / 74,3 \%)$ verificó valores satisfactorios, pero no se alcanzó la totalidad de los casos. En la mitad de los mismos la entrega de la complementación fue adecuada. La referencia y la contrarreferencia aparece con baja fre- cuencia en los registros $(22,7 \% / 20,3 \%)$ y las hojas respectivas no formaban parte de ninguna historia; criterio a ajustar, ya que incide en el nivel de atención brindada.

Los partos fueron institucionalizados en su totalidad. Un bajo porcentaje de control de puerperio $(36,9 \% / 26,2 \%)$ pone de manifiesto una desviación de la norma que puede impactar negativamente en la salud de las mujeres.

Frente a estos resultados cabe preguntarse cuáles son las causas que operan para que un programa centrado en los aspectos maternoinfantiles no logre captar a los niños para dar cumplimiento a la norma; cuáles son los motivos, desde la perspectiva de las madres, para no llevarlos o llevarlos a otros servicios y por último, cuáles son los obstáculos que operan a nivel del servicio.

Con respecto al cumplimiento de las normas y procedimientos propuestos del PROMIN, referidos a los niños desnutridos y a las embarazadas atendidos durante el año 1998, se establecieron similitudes y diferencias entre ambos centros.

En cuanto al cumplimiento verificado para los niños desnutridos en Sur se constató un mejor cumplimiento de las citaciones de seguimiento de crecimiento y desarrollo que en Policial. La inclusión de las curvas para la valoración del estado nutricional de los niños está incorporada en ambos centros.

El estado nutricional de los niños aparece más desventajoso en Sur que en Policial. Con relación a lo anterior cabe explicitar que la entrega de la complementación alimentaria apareció más desordenada en el primer centro, el interrogante es que si la situación de carencia puede actuar como factor condicionante para tal tipo de conducta traducida en los registros.

\section{De los factores contextuales (del ambiente organizativo) en el desarrollo del proyecto desde la perspectiva de los jefes de Centros de Salud y CDI}

Con respecto a los contenidos recuperados en los encuentros con los jefes de Centros de Salud, ambos percibieron como conflictivo el inicio de la gestión con PROMIN, expresaron esta valoración en nombre de los respectivos equipos de salud. Los dos entrevistados identificaron factores estructurales y organizacionales, que actuaron como obstáculos en el momento del comienzo de las acciones del programa: la falta de una buena comunicación con los equipos locales generaron situaciones de malestar entre ellos y los técnicos PROMIN, la condición de programa vertical y focalizado, la imposibi- 
lidad de participación en el momento de fijarse las condiciones de desarrollo, el criterio de focalización.

La representación del programa fue visualizada como una carga pesada, con más trabajo por lo mismo ("...sin modificación de las condiciones contractuales de trabajo"), y con interrogantes acerca de cuáles eran los beneficios para la población. Se generó mucha resistencia con la nueva forma de trabajo, el adecuarse a nuevos registros y a nuevos circuitos.

Surgieron diferencias en cuanto al proceso vivido: los jefes de Sur/CDI Gurí dieron cuenta de haber transitado por distintos momentos en el proceso de implantación de PROMIN, se deduce de sus aportes una visión positiva, pero con contenido crítico en aquellos aspectos inherentes al diseño y ejecución del programa, valoraron positivamente las mejoras logradas con respecto a la infraestructura, el aumento de la oferta de servicios y la articulación de acciones con el CDI.

En tanto la percepción de los representantes de Policial/Jardín № 37 estaría ofreciendo un registro de factores contextuales que operaron desfavorablemente para la implantación. Uno de ellos expresa una visión favorable unida a la cotidianeidad operativa del Centro de Salud, reconociendo los aportes de recursos realizados por el programa, mientras que el otro asume en sus valoraciones un posicionamiento crítico y negativo hacia el programa.

La articulación salud/desarrollo infantil, componente estructural del programa, generó vivencias casi opuestas en los jefes: en Sur se produjo un proceso de articulación facilitado por la proximidad física de ambos efectores y la coincidencia ideológica entre los equipos respectivos; en tanto que en Policial, la propuesta de integrar establecimientos de distinta dependencia, sin la mediación de los correspondientes niveles decisorios y ejecutivos, con ausencia de trabajo sostenido a nivel local para el logro del consenso, llevó al desarrollo de acciones en salud sin posibilidades de dar cuenta de la propuesta de articulación programática.

En Sur se produjo un proceso de apropiación y de flexibilización en la ejecución desde los factores contextuales identificados, tanto estructurales como organizacionales. Según la valoración vertida: “...dejó de ser 'resistido', para que el equipo comenzara a reflexionar acerca de los beneficios relativos que otorgaba, en el trabajo con los pacientes, en los registros y para la población". En Policial el programa fue asumido con cierta resistencia y la visión retrospectiva permitió dar cuenta de una adaptación limitada por la falta de articulación propuesta originalmente.
En este centro el posicionamiento asumido estuvo más en el orden de lo lineal, es decir, el equipo trabajó con un programa más, a partir del cual se obtuvieron para el centro ciertas mejoras edilicias y organizativas, la preocupación giró en torno de la sustentabilidad del programa.

En el Centro de Salud Policial el programa fue asumido con cierta resistencia, el análisis retrospectivo permitió dar cuenta de una adaptación, pero con limitaciones por la falta de articulación propuesta originalmente.

Resulta destacable la consistencia y la uniformidad de las respuestas brindadas por la directora del Jardín № 37 respecto al desacuerdo absoluto en relación a PROMIN.

La contrapartida aparece en la valoración realizada por el responsable de Gurí, quien da cuenta de una experiencia distinta, aún no negando la existencia de obstáculos.

La articulación con los CDI presentó características diferenciales entre los centros. En Sur se verificó una mejor relación aunque altamente perfeccionable. En Policial, quedó de manifiesto una débil y escasa articulación.

\section{De la accesibilidad y la aceptabilidad desde la población}

El grupo de mujeres entrevistadas compartían los siguientes rasgos socioculturales: eran en su mayoría jóvenes, predominantemente con nivel de instrucción primario, que no trabajaban fuera del hogar, integrantes de familias numerosas donde el rol de las abuelas fue recuperado por la responsabilidad que les cabía en el cuidado de los niños y, en algunos casos, como sostén del hogar; con parejas que tenían alto grado de precariedad e inestabilidad laboral, mayoritariamente asistentes a los servicios de salud públicos por no contar con cobertura de atención médica.

Este conjunto de relaciones construidas a partir de la observación de situaciones objetivas tiene un peso importante en el momento de la conformación de las representaciones en torno a la accesibilidad y aceptabilidad del programa, en los espacios de los Centros de Salud y CDI que las madres reconocen como jerárquicos.

Con respecto a la valoración del programa a partir de las representaciones construidas por las madres, se destaca el reconocimiento entre ellas de los centros como lugar de referencia para la atención, por la cercanía a sus domicilios, así como lo que ellas denominan la “buena atención" de los médicos.

Este reconocimiento es posible que se halle estrechamente vinculado con la historia de las instituciones seleccionadas, con una trayecto- 
ria de trabajo en el área desde larga data, reconociéndose claramente a los equipos profesionales como referentes de los grupos poblaciones destinatarios de sus acciones.

Pudieron dar cuenta del alcance de las prestaciones específicas de cada lugar, las posibilidades con las que cuentan, este conocimiento aparece construido desde la propia experiencia con los servicios de salud.

En sus valoraciones, las madres recuperaron explícitamente a algunos miembros del equipo de salud que cumplen un rol más significativo que el resto: como es el caso de los médicos. Respecto a ellos no enuncian mayores críticas, ya que son quienes cuidan y atienden a sus hijos y a ellas mismas en su condición de embarazadas. Se produce con estos profesionales un juego de relaciones asimétricas, que les lleva a percibir al médico en una posición de saber y de poder que no se mantiene con los restantes integrantes de los equipos de salud.

Se encontraron diferencias en la utilización y el tipo de adecuación que realiza la población con las características de los recursos de atención 22 según se trate de madres de niños desnutridos, con una periodicidad de controles marcados por el profesional según las normas programáticas y las madres de no desnutridos y embarazadas con contactos más puntuales con los efectores de salud.

Ellas llegaron a visualizar, desde su condición de usuarias de los servicios, "ciertos cambios” en la organización y atención en los centros en el transcurrir del tiempo, valoraron diferentes aspectos del proceso de acceso y utilización de los servicios traducidos en distintos grados de apropiación del efector según las experiencias con la atención y el efector; el PROMIN, sólo fue puesto en escena a partir de las intervenciones del entrevistador, la población sólo visualizó los obstáculos y/o facilitadores desde su lugar de usuario de los servicios.

Las mujeres identifican la complementación alimentaria como el auxilio ante situaciones de carencia; en este sentido la referencia a estas intervenciones la realizaron con claro contenido aprobatorio. Son los indicadores concretos para la comunidad de la presencia del programa en los servicios.

La articulación entre salud/CDI fue reconocida en Sur, mientras que en Policial lo enunciaron como un componente totalmente ajeno, visualizaron el Jardín como el inicio de la etapa escolarizada de los niños. No tienen incorporada la imagen de espacio de crecimiento integral de los niños, sólo refieren al comedor al cual se los envía, a propuesta de los médicos, en procura de alimento para sus hijos 19 .
Las prácticas profesionales, la complementación alimentaria y la relación médico paciente son los tres componentes resaltados en los comentarios que dan cuenta de la satisfacción de las madres en relación con la efectividad en el proceso de atención.

Los atributos de la atención son vistos como un encadenamiento en la cual aparecen la dimensión "trabajo" (como ausente) vinculada a la ejecución del programa y la "ayuda social" ligada al mismo.

Las madres pudieron dar cuenta de lo visible, que es lo material. En las modalidades de atención marcaron cambios que incidían en mayor medida en formas organizativas ordenadoras, por lo cual la valoración recayó casi exclusivamente en la entrega de cajas de alimentos.

\section{Del nivel de implantación logrado}

La evaluación del programa, realizada por los jefes de centros aportó, desde otra perspectiva, información que confirma y refuerza la obtenida a través del estudio de la gestión llevado a cabo por medio del análisis de los registros de los casos (centros), y fue coincidente, en algunos aspectos, con las representaciones de las madres en torno al programa.

La evidencia empírica reunida puso de manifiesto un nivel de implantación diferente en cada uno de los Centros de Salud: para Sur los condicionantes contextuales identificados por los representantes de salud y desarrollo infantil, tuvieron que ver con la programación vertical, los criterios de selectividad y la escasa adecuación de la implantación a la realidad local, que operaron como obstaculizadores en un primer momento, para luego ser superados, en forma relativa, en función del proceso de apropiación que se dieron los equipos respectivos.

En tanto, la percepción de los representantes de los centros Policial/Jardín № 37 estaría ofreciendo un registro de factores contextuales que operaron desfavorablemente en el proceso de implantación. No se produjo la articulación entre estos servicios por tratarse de instituciones con distinta dependencia funcional y administrativa y la discontinuidad de trabajo conjunto entre los equipos locales de ambas instituciones con los técnicos gerentes del programa para el logro de consenso. Estos factores obstaculizaron la posibilidad de desarrollar un planteamiento de atención profesional integrador para dar cumplimiento a las actividades propuestas por el programa en cuanto a la detección, seguimiento y contención de los niños desnutridos, reafirmándose con la visión frag- 
mentada de las acciones programáticas expresada por las madres, quienes marcaron las dificultades para el pleno acceso a los servicios.

La experiencia recogida indica que, en la medida que no se tomen en cuenta las características propias de los espacios seleccionados para ejecutar el programa, se está introduciendo un obstáculo que puede llegar a repercutir en las acciones directas con la población beneficiaria.

La calidad de los registros evaluados mostró deficiencias y escasez de precisión en los contenidos, repercutiendo en la valoración de la eficacia de los resultados. Los profesionales no jerarquizaron el registro de las prestaciones realizadas y de los hallazgos en las historias clínicas, como conducta facilitadora de la tarea asistencial para la evaluación de desempeño clínico, de gestión y de investigación, tanto desde la óptica de los servicios como de la población que accede a los servicios, por tratarse de un documento básico de su historia de saludenfermedad.

Los conjuntos sociales, en cada espacio particular, acumularon experiencias y operaron en consecuencia para resolver el proceso de salud-enfermedad-atención. Conocedores de la lógica y de los circuitos de atención pudieron hacer un mejor uso de los recursos y en consecuencia, la circulación dentro de los servicios también se vio facilitada 20 .

La gestión del programa realizado por los equipos técnicos transitó (en Sur) por distintos momentos caracterizados por una mayor o menor flexibilidad en las relaciones entre los equipos locales y los responsables. Se recuperó el diálogo como medio de intercambio de ideas y como favorecedor de la gestión para plantear diferencias, necesidades y posibilidades; en definitiva, para poder construir y lograr un mejor nivel de implantación.

\section{Discusión}

La evaluación del PROMIN, fue planteada con la intencionalidad de identificar los condicionantes que operaron en la puesta en marcha de PROMIN, poniendo en juego tres niveles de análisis: los factores contextuales percibidos por los jefes de Centros de Salud y jefes de CDI que condicionaron la puesta en marcha del programa; la gestión desde la eficacia de los resultados obtenidos en la utilización por una parte de la población beneficiaria (niños desnutridos y embarazadas), en términos de la concordancia entre lo recomendado y lo realizado, y las representaciones de la población en torno a la accesibilidad, aceptabilidad y satisfacción de PROMIN.

A partir de este marco es posible concluir que el nivel de implantación logrado por PROMIN se manifestó a través de la relación entre los factores contextuales que condicionaron las estrategias de intervención desarrolladas desde los servicios, las que a su vez se interrelacionaron con las representaciones construidas desde la población.

En términos generales la evaluación del programa, realizada por los cuatro jefes de Centros de Salud y de CDI aportó, desde otra perspectiva, información que confirma y refuerza la obtenida a través del estudio de la gestión llevado a cabo por medio del análisis de los registros de los centros, y fue coincidente, en algunos aspectos, con las representaciones de las madres en torno al programa.

La evidencia empírica reunida pone de manifiesto que el nivel de implantación de PROMIN no resultó homogéneo en los dos centros tomados en consideración.

De las expresiones vertidas por los jefes de centros entrevistados, se recupera como lo más destacado la necesidad que tuvieron los equipos técnicos pertenecientes a la estructura programática de flexibilizar criterios y considerar la realidad local particular, ya que un posicionamiento más estratégico por parte de los mismos, podría haber facilitado la implantación adecuada de la intervención.

La evaluación de la gestión desde la eficacia de los resultados obtenidos en la utilización por parte de los desnutridos y las embarazadas, en términos de la concordancia entre lo recomendado y lo realizado, desde los registros realizados por los integrantes de los equipos de salud de los centros, plantea serias dudas respecto del cumplimiento ajustado de las normas y procedimientos propuestos por el programa, con algún grado de variación según se trate de uno u otro centro y de la actividad realizada.

Si se evalúa la eficacia del programa, mediado por los registros realizados en función de las normas, no podría ser calificado como satisfactorio. Se impone la necesidad de revisar los criterios de registro y la jerarquización de la documentación clínica en búsqueda de un mejor desempeño y atención de calidad.

Ambos centros reconocieron elementos positivos y negativos en su desempeño. Las normas no fueron cumplidas en su totalidad en todos los aspectos tenidos en cuenta en esta investigación, lo que significaría la necesidad de una puesta a punto de las mismas en función de las realidades encontradas, pero desde el posicionamiento servicios/población. 
El comportamiento presentado en los dos centros no es igual, como tampoco es igual la relación establecida entre los dos efectores (Centros de Salud y CDI) articulados por PROMIN.

Se pone en discusión la eficacia de la propuesta en términos de la recuperación de los niños y hasta qué punto estas medidas parciales son útiles para aliviar situaciones de profunda crisis no resolviendo los problemas de fondo.

Con relación a las representaciones verbalizadas por las madres entrevistadas, las mismas delinean una construcción común, recuperan y reconocen la proximidad de los centros de la vecindad y aceptabilidad con la atención brindada. Se trata de servicios con gran notoriedad local; en este punto el programa implementado, no introdujo variaciones significativas en cuanto a la posibilidad de modificar esta representación, sí se reconocen algunas instancias de ordenamiento para la realización efectiva de los controles y de la organización de algunos aspectos que ocasionan una mejor utilización de los servicios, con el agregado de los beneficios recientemente incorporados.

La percepción que las madres tienen de los respectivos CDI es diferencial, el primer eje diferenciador lo constituye el espacio desde el cual emiten su opinión, o sea, centro de salud Sur o Policial y el segundo eje son los valores puestos en juego en las reflexiones realizadas. En algunas oportunidades se cuestiona el esquema organizacional del CDI.

Se hace evidente que los dos servicios de salud tomados en cuenta aparecen con una trayectoria reconocida por estas mujeres y, justamente por ello, el programa aparece como una intervención que no ha modificado la forma de ejercicio ni de contención ejercida por los profesionales que en ellos se desempeñan, más allá de la relación asimétrica que queda planteada en todo momento.

Los profesionales se constituyen en referentes claros de la población antes y durante el desarrollo del programa cumpliendo un rol de "facilitadores" en la mayoría de los casos.

Puede concluirse que toda transformación institucional es válida, en tanto tenga un enfoque y un diseño organizacional orientado a ofrecer un servicio para satisfacer las necesidades de los niños en riesgo, la misma debe complementarse con el logro de una legitimación por parte de los grupos hacia quienes están dirigidas las acciones.

\section{Consideraciones finales}

De acuerdo a los resultados hasta aquí presentados y discutidos, caben algunas consideraciones finales.

En la evaluación de programas debe quedar explicitado que ninguna medida aislada resulta en una justa estimación del grado de implantación de una intervención. Se hace necesario abordar metodologías combinadas, a efectos de dar cuenta, en forma más ajustada y precisa, de los resultados e impactos logrados en la población destinataria de tales programas sociales.

Una de las formas posibles sería la ampliación de la indagación con la incorporación de otros integrantes de los equipos de salud que participaron, como forma de conocer las valoraciones de otros actores. También dado el tiempo transcurrido, sería oportuno abordar la búsqueda de la medición del impacto logrado por el programa en aquellos niños desnutridos recuperados que concurrieron a los CDI en términos de los logros, en términos de conocimientos, habilidades y prácticas, comparándolo con aquellos que no concurrieron.

El supuesto de partida fue que la validez externa de los resultados hasta aquí presentados podrá probarse en la medida que se llegue a pensar, que en los espacios de los restantes servicios bajo programa se producen realidades similares a las aquí presentadas.

La implantación de un programa es un proceso de negociación y de consenso entre los distintos actores y debe sensibilizarse a los políticos que toman decisiones para estimular el trabajo desde el nivel local, ya que son quienes conocen la realidad que debe ser modificada.

Si la articulación de los equipos se produce antes que la ejecución de las intervenciones, se garantizará parte del éxito futuro.

Los programas de corte asistencial sólo llegan a encubrir en parte los cuadros de situación en los que desarrollan sus vidas grandes grupos poblacionales, donde el estado nutricional deficitario de los menores es uno de los indicadores de máxima vulnerabilidad. Las "soluciones" coyunturales incluidas en estos programas sólo son pinceladas, el círculo de la pobreza y de la mal nutrición no acaba. 


\section{Resumen}

Se presenta la evaluación del Programa Materno Infantil y Nutrición (PROMIN), dirigido a embarazadas y niños menores de cinco años, ejecutado en el Municipio de Rosario, Argentina. El objetivo es identificar los condicionantes que operaron en la puesta en la marcha según: el ambiente organizacional percibido por los jefes de los Centros de Salud y Desarrollo Infantil; la gestión de las actividades de los equipos de salud y las representaciones de la población en torno a la accesibilidad y aceptabilidad con el programa. Se seleccionaron como casos, dos servicios de salud bajo programa durante 1998. Se utilizaron estrategias cuantitativas y cualitativas. Los resultados indican que el

\section{Referencias}

1. Sistema de Información, Monitoreo y Evaluación de Programas Sociales/Organización de las Naciones Unidas para la Educación, la Ciencia y la Cultura. Gestión integral de programas sociales orientada a resultados. Manual metodológico para la planificación y evaluación de programas sociales. Buenos Aires: Fondo de Cultura Económica; 1999.

2. Tanaka O, Cesar CL. Inquérito domiciliar como instrumento de avaliação de serviços de saúde: um estudo de caso na região sudoeste da área metropolitana de São Paulo, 1989-1990. Cad Saúde Pública 1996; 12 Suppl 2:59-70.

3. Contandriopoulos A, Champagne F, Denis J, Pineault $R$. L'evaluation dans le domaine de la santé: concepts et methods. In: Lebrun T, Sailly JC, Amouretti L. L'evaluation en matière de santé: des concepts à la pratique. Lille: Cresge; 1992. p. 14-32.

4. Hartz Z. Evaluation du programme de santé maternelle et infantile dans une región du Nord-Est du Brasil [Tesis Doctoral]. Montreal: Université de Montreal; 1993.

5. Chen H-T. Theory - driven evaluations. Thousand Oaks: Sage Publications; 1990.

6. Denis JL, Champagne F. Analyse d'implantation. Montreal: Groupe de Recherche Interdisciplinaire en Santé, Université de Montréal; 1990.

7. Patton M Q. Utilization-focused evaluation. 2nd Ed. Beverly Hills: Sage Publications; 1986.

8. Ministerio de Salud y Acción Social de la Nación. Proyecto materno infantil y nutrición. Documento de trabajo. Buenos Aires: Ministerio de Salud y Acción Social de la Nación; 1992.

9. Donabedian A. La calidad de la atención médica. México DF: La Prensa Mexicana; 1984.

10. Jodelet D. Las representaciones sociales: un área en expansión. In: Páez D, San Juan C, Romo I, Vergara A, editores. Sida: imagen y prevención. Madrid: Editorial Fundamentos; 1991. p. 25-56.

11. Aday LA, Andersen R. Marco teórico para el estudio del acceso a la atención médica. In: White KL, ambiente organizacional condicionó diferencialmente las estrategias de intervención en ambos centros. Las coberturas alcanzadas fueron diferentes en ambos centros, con una meta del 80\%. El registro de las actividades por parte de los equipos da cuenta de un cumplimiento parcial y heterogéneo en ambos espacios. Las madres reconocen las instituciones por su prestigio, conocen el alcance de las prestaciones y de los servicios más allá de PROMIN. La aceptabilidad se expresó como la provisión de complementación alimenicia.

Política Social; Evaluación de Programas; Salud Materno-infantil editor. Investigaciones sobre servicios de salud: una antología. Washington DC: Organización Panamericana de la Salud; 1992. p. 604-13. (Publicación Científica, 534).

12. Yin RK. Applications of case study research. Newbury Park: Sage Publications; 1993.

13. Yin RK. Case study research: design and methods. Thousand Oaks: Sage Publications; 1984. (Applied Social Research Methods Series, 5).

14. Kessner DM, Kalk CE, Sniger J. Assessing health quality. The case of tracers. N Engl J Med 1973; 288:189-94.

15. Forni FH, Gallart MA, Vasilachis de Gialdino I. Métodos cualitativos II. La práctica de la investigación. Buenos Aires: Centro Editor de América Latina; 1993.

16. Dever G, Champagne F. Epidemiology in health services management. Rockville: Aspen Systems Corporation; 1984.

17. Frenk J. El concepto y la medición de la accesibilidad. In: White KL, editor. Investigaciones sobre servicios de salud: una antología. Washington DC: Organización Panamericana de la Salud; 1992. p. 929-43. (Publicación Científica, 534).

18. Minayo MCS. O desafío do conhecimento. Pesquisa qualitativa em saúde. 4a Ed. São Paulo: Editora Hucitec/Rio de Janeiro: ABRASCO; 1996.

19. Garrote N. Algunas reflexiones acerca de la contribución de la antropología a la problemática de la alimentación y la salud. Investigación en Salud. Publicación Científica de la Secretaría de Salud Pública Municipal 2000; 3:131-40.

20. Whitaker-Dalmaso A, Senna D. Desenvolvimento de alternativas para recepção da demanda espontânea em unidade básica de saúde. Saúde Debate 1995/1996; 49-50: 83-7.

Recibido el 23/Jun/2004

Versión final presentada el 25/May/2005

Aprobado el 14/Jul/2005 